\title{
Design, implementation and self-tuning adaptive control of maglev guiding system
}

\author{
Mei-Yung Chen ${ }^{\mathrm{a}, *}$, Kun-Nan $\mathrm{Wu}^{\mathrm{a}}$, Li-Chen $\mathrm{Fu}^{\mathrm{a}}$, b \\ ${ }^{a}$ Department of Electrical Engineering, National Taiwan University, Taipei, Taiwan \\ ${ }^{\mathrm{b}}$ Department of Computer Science and Information Engineering, National Taiwan University, Taipei, \\ Taiwan
}

Received 27 October 1998; accepted 13 May 1999

\begin{abstract}
This paper presents a self-tuning adaptive control (STA control) for a magnetically levitated (maglev) guiding system. The guiding system is a repulsive maglev system with a passive carrier and four active guiding tracks. Hybrid magnets exert the levitation forces, whereas the stabilizing forces are produced by electromagnets. Given such a system, an STA stabilizing controller is developed, and the thorough analysis of the stability property is also proposed. It is shown that without precise knowledge of various components, the overall system stability and the regulating precision are assured, which validates the hereby proposed system. From the simulation and experimental results, the performance of the system design and the enforced control mechanism is demonstrated successfully. (C) 1999 Elsevier Science Ltd. All rights reserved.
\end{abstract}

Keywords: Maglev; Hybrid magnet; Self-tuning adaptive control

\section{Introduction}

Recently, magnetic levitation is considered as one of the most suitable ways to achieve high precision transportation. According to Hollis et al. [1,2], it creates a stable state without any mechanical contact when the gravitational force is solely

\footnotetext{
* Corresponding author.

E-mail address: cmy@cc.ee.ntu.edu.tw (M.-Y. Chen).
} 
counterbalanced by magnetic forces. Of course, such contact-free levitation should hold for all degrees of freedom of the rigid body.

Often, a distinction is made between magnetic suspension and magnetic levitation. The former refers to systems subject to attractive magnetic forces whereas the latter to those subject to repulsive ones. However, the strict meanings of the two terms fail to embrace all types of contact-free magnetic support. Therefore, it is the trend to use the term 'levitation' in a more general sense, and to abbreviate "magnetic levitation" to "maglev".

Previous work in maglev systems spans many fields. A large volume of literature has been published. Some well known fields include maglev transportation [3,4], wind tunnel levitation [5], magnetic bearings [6], and antivibration tables [7]. Here, however, we will only investigate the maglev techniques for the field of short-range travel with precision positioning, and then design and implement a prototype maglev system to verify its high performance.

In general, maglev systems can be classified into two categories according to whether the utilized magnetic forces are attractive or repulsive, each with various kinds of possible arrangement as shown in Table 1.

From Table 1, we can observe that the arrangements A1, A2, and R1 need onboard batteries. Except for arrangement R2, almost all the maglev setups place their control circuits onboard the carrier. However, not only batteries but also electronic elements onboard will increase the weight of the carrier, and further increase the power consumption for completing the levitation.

Notably, the weight of the carrier in maglev system R2 is counterbalanced by the magnetic force generated by the opposing magnetic fields between the permanent magnets on the carrier and the electromagnets inside the guiding tracks, so that no power consumption is required for the carrier levitation. But, unlike the attractive case, a repulsive maglev is vertically stable, but laterally unstable. Therefore, an appropriate mechanical design with additional electromagnets should be necessary to provide proper guiding control of the carrier. A prototype maglev silicon wafer transporter, designed by Wang [8], is an example which applied the aforementioned technique.

On account of these factors, permanent magnets are preferably used in our maglev system for levitation, and electromagnets are then used for positioning and stabilization. In this paper, a prototype maglev guiding system, which takes a

Table 1

Comparison of maglev systems

\begin{tabular}{lllll}
\hline Maglev system & Index & Type of magnets & Battery on carrier & Controllability \\
\hline Attractive & A1 & DC electromagnet & large size & good \\
& A2 & Hybrid magnet & medium size & fair \\
\multirow{2}{*}{ Repulsive } & A3 & AC electromagnet & no & good \\
& R1 & DC electromagnet & medium & good \\
& R2 & Permanent magnet & no & poor \\
\hline
\end{tabular}


concept similar to [8] but is its improved version, is designed and implemented. Based on this system, an STA controller is proposed and thoroughly analyzed for system stabilization property. Extensive simulation and experimental results are provided to demonstrate the feasibility and effectiveness of the developed system and designed controller.

The organization of this paper is as follows. Section 2 describes the design aspects of the hereby implemented prototype system. In Section 3, detailed mathematic modeling is provided. Section 4 develops the STA controller for the prototype maglev system. Section 5 presents extensive simulation and experimental results to demonstrate the effective use of the system design and its controller. Some discussion is also provided in Section 5. Finally, conclusions are drawn in Section 6

\section{Prototype system design}

Fig. 1 shows the directions of force and torque exerted on a single dipole moment due to a current-carrying straight wire. Currents in opposite directions are considered in the figure, and the dipole moment is upward and located at eight different locations around the wire.

The idea of a maglev four-track guiding system can be traced back to [8]. In this paper, we adopt that idea and propose a variant design, which bears several improvements on [8]. Distinct features will be discussed in Section 5.

For application of a short-range travel with precision positioning, the maglev system to be designed should meet the following requirements:

1. The system should constrain motions of the movable carrier to provide the desired degree of freedom for the mounted actuator, e.g., a linear motor.

2. The system performance should not be changed regardless of the location of the levitated carrier within the entire traveling range.

3. When the stabilization controller ceases to work, the damage should be kept to the minimum.

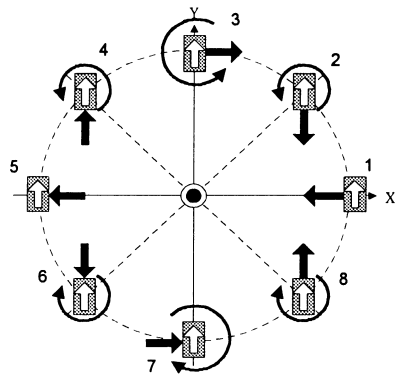

(a)

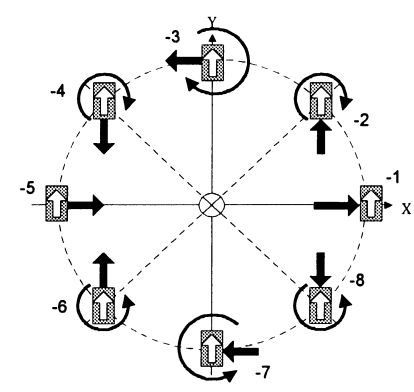

(b) $\otimes$ Inward Current

Fig. 1. Forces and torques on a single dipole moment due to a current-carrying straight wire. 
4. The load capacity of the carrier should be at least $0.5 \mathrm{~kg}$ with $20 \mathrm{~cm}$ travel distance to meet the application to wafer transportation.

\subsection{Passive carrier and active track}

In the system with passive carrier riding on the active tracks, the power supply and control module are no longer fixed on the carrier so that its overall weight can be reduced considerably, and hence the power needed to levitate the carrier can be rather economical. As such, a compact maglev system may become much more realizable. Moreover, the high precision requirement may be accomplished more easily under this kind of arrangement.

In order to produce a uniform magnetic field, an oblong coil is necessary, as illustrated in Fig. 2. In this case, the magnetic force applied to the permanent magnet will be smaller than that in the former for the sake of its lower field gradient. But fortunately, permanent magnet within the coils can provide a major levitation force. Therefore, it seems that this oblong coil with a permanent magnet becomes the most appropriate choice to meet our special purpose.

For a free-floating system, the total six DOFs of the carrier should be taken into consideration, namely, $X, Y, Z, \varphi, \phi$, and $\theta$. Their definitions are shown in Fig. 3, where $Y$ is the moving direction and $Z$ is the floating height of the carrier. As has been mentioned earlier, the repulsive maglev system is vertically stable, and hence the $Z$ direction is always a stable mode. However, there is no constraint force in the $Y$ direction for the positioning requirement. Therefore, we should only concern the rest of four DOFs, $X, \varphi, \phi$, and $\theta$ which are those undesirable DOFs in our free-floating construction.

In order to simplify the overall controller design, a mechanism is better devised such that each of the four above DOFs can have an independent control. Therefore, a four-track concept is adopted based on the following philosophy (see Figs. 4 and 5). First, a pair of tracks can levitate a carrier while stabilizing $\phi$, such as the rail of a train, but the variable $\varphi$ cannot be controlled when the weight of the carrier is not uniformly distributed, as shown in Fig. 4. Therefore, another pair of tracks are added to create the controllability of the angle $\varphi$. Such fourtrack arrangement is somewhat like the four wheels of an automobile as shown in Fig. 5. As a result, we have reduced the undesirable DOFs to only $X$ and $\theta$. Intuitively, in order to control the modes $X$ and $\theta$, additional electromagnets will

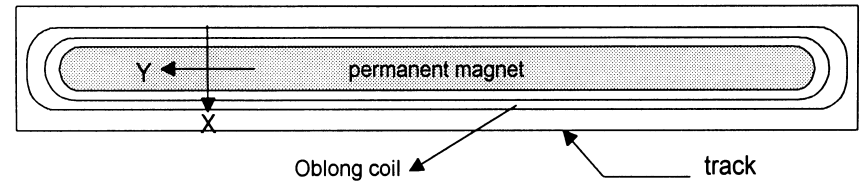

Fig. 2. Oblong coil. 


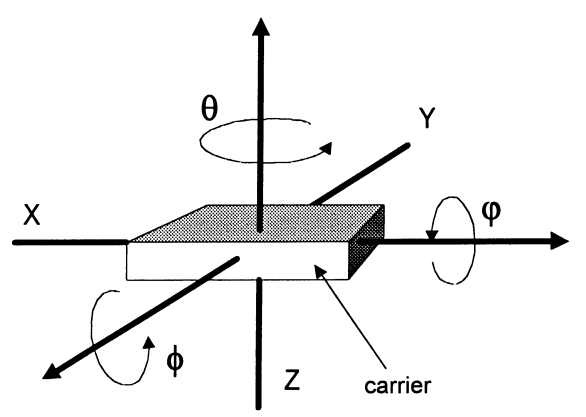

Fig. 3. Body coordinate of the carrier.

have to be set up in compliance with the affixture of four permanent magnets on the carrier.

\subsection{Stabilizer design}

Via the symmetric design of the carrier as well as the four levitation tracks, only two modes, $X$ and $\theta$ are left to be controlled, which implies that only an in-plane motion stabilizer needs to be designed in our setup. Therefore, the function of the stabilizer is to provide lateral pull or push forces exerting on the magnets of the carrier as shown in Fig. 6.

Referring to Fig. 1, when a magnet with upward dipole moment is placed at positions (1), (5), (-1) or $(-5)$, the magnet will merely experience a lateral magnetic force, which forms the underlying working principle of our stabilizer. However, from the viewpoint of control, the two stabilizers should be separated as far as possible so that the two respective controls will not interfere with each other.

In Fig. 6, the large arrows denote the desired control forces exerting on the magnets when the carrier underwent $\theta$ rotations in Fig. 6 (a) and (b), and $X$ translations in Fig. 6(c) and (d). It is obvious that the inner forces are always in the same direction in all the situations, and so are the outer forces. This observation implies that two independent inputs are sufficient to handle the control of the in-plane motion. Therefore, if the wires of the two inner coils as

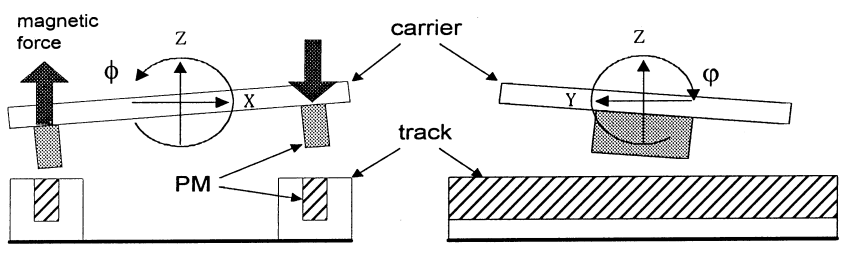

Fig. 4. Two-track concept. 

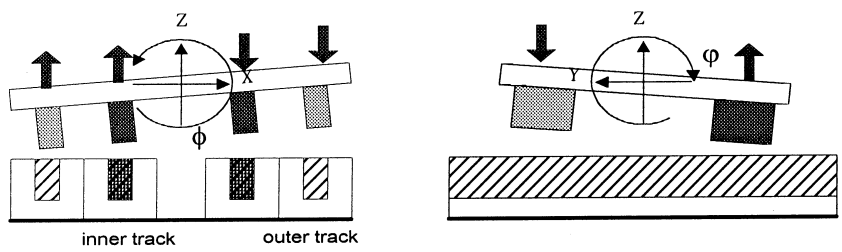

Fig. 5. Four-track concept.

well as those of the two outer coils are respectively connected, the aforementioned controllability is established.

\subsection{Sensor system design}

For subsequent controller design, focus will be placed on the lateral stabilizing control since $(X, \theta)=(0,0)$ is an open-loop unstable equilibrium point. Accordingly, design of a lateral sensor system will also be our focus in order to establish the control.

Fig. 7 shows the setup of our optical sensing system. There are two laser emitters along with two photodetectors arranged in parallel with the levitation tracks. Two shielding plates are mounted on the two corners of the carrier in order to block the two collimated laser beams. Since the output voltages of the

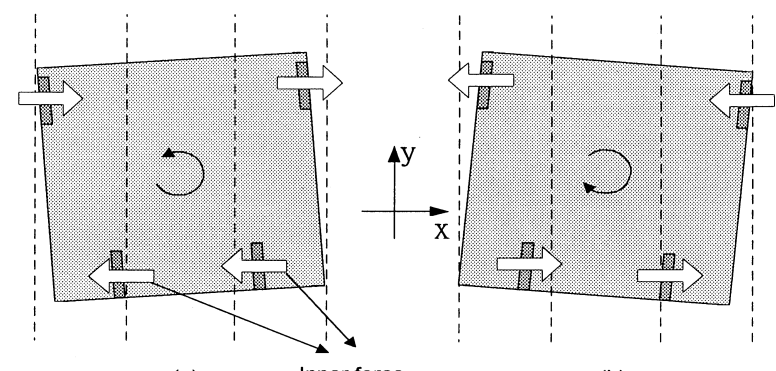

(a)

(b)

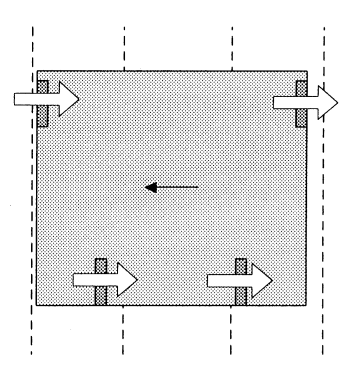

(c)

outer force

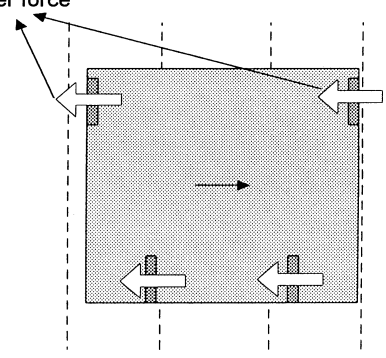

(d)

Fig. 6. Control forces acting on the magnets when the carrier underwent some rotations and translations. 


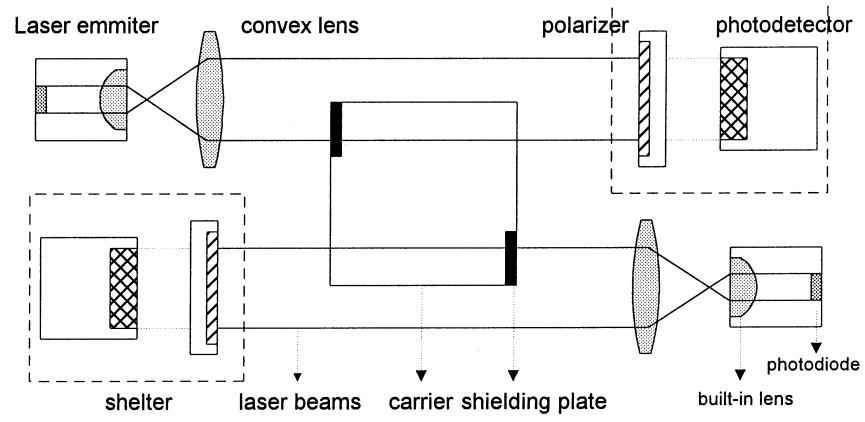

Fig. 7. Final integration in the optical sensing system.

photodetectors are proportional to the amounts of laser beam incident on respective sensing areas, the data of the carrier's translation and rotational displacements can be obtained through proper signal processing of the two signals.

Since the voltage magnitudes $V_{1}$ and $V_{2}$ from the photodetectors are directly proportional to the lateral displacement of the shielding plates, respectively, the translation displacement of the carrier can be expressed as [8]:

$$
X=\frac{K}{2}\left(V_{1}-V_{2}\right)
$$

where $K$ is the constant relating the signal voltage to the displacement of the shielding plate. On the other hand, after assigning the output voltages $V_{0}$ when the carrier is at the neutral point 0 , we can obtain the expression of the rotational displacement $\theta$ as:

$$
\theta=\frac{K}{2 r \cos \left(\theta_{0}\right)}\left(V_{1}+V_{2}\right)
$$

where $r$ is the distance from carrier center to the corner and $\theta_{0}$ is the nominal angle.

So far, we have discussed two typical situations, one with the carrier for pure translational displacements and the other for pure rotational displacements. For

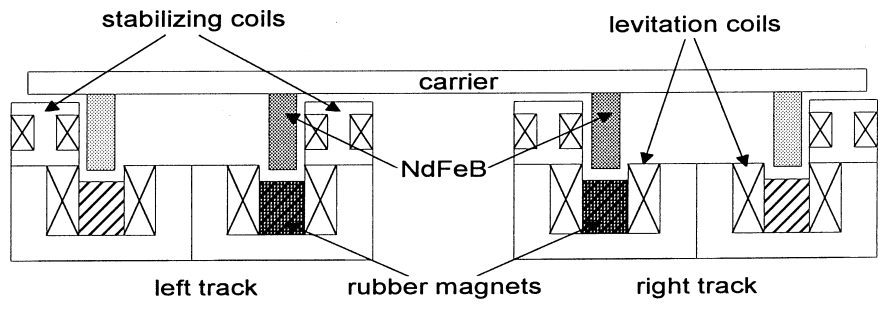

Fig. 8. Overall four-track system. 
the hybrid situations, we note that Eqs. (1) and (2) can both be applied to the entire $X-\theta$ domain because they are linearly independent. Therefore, the working principles of our optical sensing system for the purpose of stabilizing control are now justified.

The carrier shape is a cuboid with dimensions $200 \times 200 \times 10 \mathrm{~mm}$, and the total mass of the carrier without load is $643 \mathrm{~g}$. The length of each levitation track is $40 \mathrm{~cm}$ and its width is $35 \mathrm{~mm}$. The overall system arrangement is shown in Fig. 8.

\section{System modeling}

From the previous discussion, stabilization of the entire maglev system is in fact a two-input two-output system.

In Fig. 9, we let the carrier undergo a translation of $X$ and a rotation of $\theta$. Here, the coordinate frame $X Y Z$ denotes the global inertial coordinate system with $Z$ pointing out of paper. The local coordinate of the carrier, $x y z$, is chosen to be coincident with the carrier's principal axes so that the products of inertia are identically zero. Furthermore, we assume the forces applied to the magnets attached onboard are concentrated at their centroids.

To obtain the equations of motion for a rigid body, we have:

$$
\begin{aligned}
& \Sigma F=m \ddot{X}, \\
& \Sigma T=J \ddot{\theta} .
\end{aligned}
$$

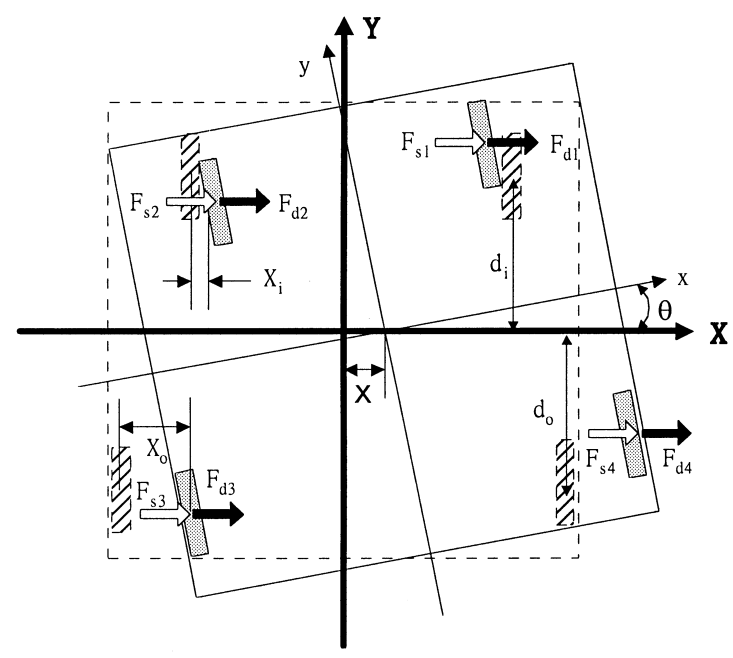

Fig. 9. The carrier undergoes $X$ translation and $\theta$ rotation. 
where $F$ and $T$ designate the external force and torque, whereas $m$ and $J$ designate the mass and the inertia with respect to the $z$ axis of the carrier, respectively.

According to Fig. 9, Eqs. (3) and (4) can be expressed as follows:

$$
\begin{aligned}
& m \ddot{X}=\sum_{i=1}^{4} F_{\mathrm{di}}+\sum_{i=1}^{4} F_{\mathrm{s} 1} \\
& \ddot{J}=-\left(F_{\mathrm{d} 1}+F_{\mathrm{s} 1}+F_{\mathrm{d} 2}+F_{\mathrm{s} 2}\right) d_{\mathrm{i}}+\left(F_{\mathrm{d} 3}+F_{\mathrm{s} 3}+F_{\mathrm{d} 4}+F_{\mathrm{s} 4}\right) d_{\mathrm{o}}
\end{aligned}
$$

where $F_{\mathrm{d} 1}$ and $F_{\mathrm{d} 2}$ are the destabilizing forces applied to the outer magnets, and $F_{\mathrm{d} 3}$ and $F_{\mathrm{d} 4}$ are applied to the inner magnets; $F_{\mathrm{s} 1}, S_{\mathrm{s} 2}, F_{\mathrm{s} 3}$, and $F_{\mathrm{s} 4}$ are the stabilizing forces defined in the same manner; $d_{\mathrm{i}}$ and $d_{\mathrm{o}}$ are the distances from the centers of the inner and outer magnets to the $X$ axis, respectively, when the local coordinate $x y z$ is coincident with the global coordinate $X Y Z$.

In the previous section, we have mentioned that the two inner stabilizing coils as well as the two outer stabilizing coils are respectively connected so that we may further simplify Eqs. (5) and (6) by letting $F_{\mathrm{s} 1}=F_{\mathrm{s} 2} \equiv F_{\mathrm{so}}, \quad F_{\mathrm{s} 3}=F_{\mathrm{s} 4} \equiv F_{\mathrm{s} \text { i }}$, $F_{\mathrm{d} 1}=F_{\mathrm{d} 2} \equiv F_{\mathrm{do}}$, and $F_{\mathrm{d} 3}=F_{\mathrm{d} 4} \equiv F_{\mathrm{di}}$.

Then, we can obtain the following resulting system:

$$
\begin{aligned}
& m \ddot{X}=2\left(F_{\mathrm{si}}+F_{\mathrm{so}}+F_{\mathrm{di}}+F_{\mathrm{do}}\right), \\
& \ddot{\theta}=-2\left(F_{\mathrm{di}}+F_{\mathrm{si}}\right) d_{\mathrm{i}}+2\left(F_{\mathrm{do}}+F_{\mathrm{so}}\right) d_{\mathrm{o}} .
\end{aligned}
$$

On the other hand, the expression of the displacements of the magnets, $X_{\mathrm{i}}$ and $X_{\mathrm{o}}$, for the inner and outer magnets, respectively, are:

$$
\begin{aligned}
& X_{\mathrm{i}}=X-d_{\mathrm{i}} \sin (\theta), \\
& X_{\mathrm{o}}=X+d_{\mathrm{o}} \sin (\theta)
\end{aligned}
$$

by referring to Fig. 6 . Since $\theta$ is a small angle of rotation so that we may replace $\sin (\theta)$ by $\theta$ and then approximate the destabilizing forces as

$$
F_{\mathrm{d}}=K_{\mathrm{d}} X .
$$

Therefore, by using Eqs. (9)-(11), the expression of the two destabilizing forces are:

$$
\begin{aligned}
& F_{\mathrm{di}}=K_{\mathrm{d}}\left(X-d_{\mathrm{i}} \theta\right), \\
& F_{\mathrm{do}}=K_{\mathrm{d}}\left(X+d_{\mathrm{o}} \theta\right)
\end{aligned}
$$

whereas the stabilizing forces are expressed as:

$$
F_{\mathrm{si}}=-K_{\mathrm{s}} V_{\mathrm{i}}
$$




$$
F_{\mathrm{so}}=-K_{\mathrm{s}} V_{\mathrm{o}}
$$

where $V_{\mathrm{i}}$ and $V_{\mathrm{o}}$ are the control voltages applied to the inner and outer stabilizers, respectively. Then, with the substitution of Eqs. (12)-(15), Eqs. (5) and (6) become:

$$
\begin{aligned}
& \ddot{X}-\frac{4 K_{\mathrm{d}}}{m} X+\frac{2 K_{\mathrm{d}}\left(d_{\mathrm{i}}-d_{\mathrm{o}}\right)}{m} \theta=-\frac{2 K_{\mathrm{s}}}{m}\left(V_{\mathrm{i}}+V_{\mathrm{o}}\right), \\
& \ddot{\theta}+\frac{2 K_{\mathrm{d}}\left(d_{\mathrm{i}}-d_{\mathrm{o}}\right)}{J} X-\frac{2 K_{\mathrm{d}}\left(d_{\mathrm{i}}^{2}+d_{\mathrm{o}}^{2}\right)}{J} \theta=\frac{2 K_{\mathrm{s}}}{J}\left(d_{\mathrm{i}} V_{\mathrm{i}}-d_{\mathrm{o}} V_{\mathrm{o}}\right) .
\end{aligned}
$$

Now, let the states be $X, \dot{X}, \theta$ and $\dot{\theta}$, and the outputs be $X$ and $\theta$, then we can compactly express Eqs. (16) and (17) in state space form as:

$$
\begin{aligned}
& \dot{\mathbf{x}}=\mathbf{A x}+\mathbf{B u}, \\
& \mathbf{y}=\mathbf{C x} .
\end{aligned}
$$

where

$$
\mathbf{x}=\left[\begin{array}{c}
x_{1} \\
x_{2} \\
x_{3} \\
x_{4}
\end{array}\right]=\left[\begin{array}{c}
X \\
\dot{X} \\
\theta \\
\dot{\theta}
\end{array}\right], \quad \mathbf{y}=\left[\begin{array}{c}
X \\
\theta
\end{array}\right], \quad \mathbf{u}\left[\begin{array}{l}
V_{\mathrm{i}} \\
V_{\mathrm{o}}
\end{array}\right]
$$

and

$$
\begin{aligned}
\mathbf{A} & =\left[\begin{array}{llll}
0 & 1 & 0 & 0 \\
\frac{4 K_{\mathrm{d}}}{m} & 0 & \frac{2 K_{\mathrm{d}}\left(d_{\mathrm{i}}-d_{\mathrm{o}}\right)}{m} & 0 \\
0 & 0 & 0 & 1 \\
\frac{2 K_{\mathrm{d}}\left(d_{\mathrm{i}}-d_{\mathrm{o}}\right)}{J} & 0 & \frac{2 K_{\mathrm{d}}\left(d_{\mathrm{o}}^{2}+d_{\mathrm{i}}^{2}\right)}{J} & 0
\end{array}\right], \quad \mathbf{B}=\left[\begin{array}{ll}
0 & 0 \\
-\frac{2 K_{\mathrm{s}}}{m} & -\frac{2 K_{\mathrm{s}}}{m} \\
0 & 0 \\
\frac{2 K_{\mathrm{s}} d_{\mathrm{o}}}{J} & -\frac{2 K_{\mathrm{s}} d_{\mathrm{i}}}{J}
\end{array}\right], \\
\mathbf{C} & =\left[\begin{array}{llll}
1 & 0 & 0 & 0 \\
0 & 0 & 1 & 0
\end{array}\right] .
\end{aligned}
$$

So far, we have obtained the system dynamics including the two unstable modes along with the two control inputs. It is a model of two-input two-output system with four states. From the system matrix $\mathbf{A}$, it is obvious that if $d_{\mathrm{i}}$ is exactly equal to $d_{\mathrm{o}}$, then $X$ and $\theta$ are decoupled. In such an ideal case, two eigenvalues of matrix $\mathbf{A}$ are positive, which in turn verifies the unstable nature of the levitation system in the physical world. 


\section{Controller design}

In general, if the on-line system identification can be achieved, the performance of the STA control should be better than that of the conservative robust control, especially in the case of a definite model structure with unknown parameters and variable loads. Nowadays, high speed PC-based controllers are more and more economical and reliable for intensive computations to realize a complex adaptive control scheme. This, therefore, motivates us to adopt an STA controller for the task of lateral stabilization.

\subsection{Plant model}

First of all, let us recall the equations of motion. In state-space form, the plant model is expressed in Eq. (18). Furthermore, by replacing all the coefficients by matrix entries, we can rearrange the plant model more conveniently. By this we can find that the model has a special regularity. Both of the matrices $\mathbf{A}$ and $\mathbf{B}$ have four entries related to the system parameters, and are all on the 2nd and the 4th rows. Such a property allows us to rewrite the first-order equations into second-order ones with only two variables:

$$
\left[\begin{array}{l}
\ddot{x}_{1} \\
\ddot{x}_{3}
\end{array}\right]=\left[\begin{array}{ll}
a_{21} & a_{23} \\
a_{41} & a_{43}
\end{array}\right]\left[\begin{array}{l}
x_{1} \\
x_{3}
\end{array}\right]+\left[\begin{array}{ll}
b_{21} & b_{22} \\
b_{41} & b_{42}
\end{array}\right]\left[\begin{array}{l}
u_{1} \\
u_{2}
\end{array}\right]
$$

Furthermore, we let the new second-order differential equations be expressed in a compact form as:

$$
\ddot{\mathbf{e}}=\mathbf{A}_{\mathbf{e}} \mathbf{e}+\mathbf{B}_{\mathbf{e}} \mathbf{u}
$$

where $\mathbf{e}, \mathbf{A}_{\mathbf{e}}$ and $\mathbf{B}_{\mathbf{e}}$ are defined as:

$$
\mathbf{e}=\left[\begin{array}{l}
e_{1} \\
e_{2}
\end{array}\right]=\left[\begin{array}{l}
x_{1} \\
x_{3}
\end{array}\right], \quad \mathbf{A}_{\mathbf{e}}=\left[\begin{array}{ll}
a_{21} & a_{23} \\
a_{41} & a_{43}
\end{array}\right], \quad \mathbf{B}_{\mathbf{e}}=\left[\begin{array}{ll}
b_{21} & b_{22} \\
b_{41} & b_{42}
\end{array}\right] .
$$

So far, we have obtained a second-order dynamic model with two variables and two compact system matrices, each of which merely contains four system-related parameters.

\subsection{Control law}

With the help of Eq. (20), it is intuitive to design a control law as:

$$
\mathbf{u}=\mathbf{B}_{\mathbf{e}}^{-1}\left(-\mathbf{A}_{\mathbf{e}} \mathbf{e}-\lambda_{1} \dot{\mathbf{e}}-\lambda_{2} \mathbf{e}\right)
$$

if $\mathbf{A}_{\mathbf{e}}$ and $\mathbf{B}_{\mathbf{e}}$ are already known, where $\lambda_{1}$ and $\lambda_{2}$ are both positive numbers.

As for the invertibility of matrix $\mathbf{B}_{\mathbf{e}}$, by Eq. (18) it is obvious that $\mathbf{B}_{\mathbf{e}}$ is in fact always nonsingular. Next, by substituting Eq. (21) into Eq. (20), we can obtain 


$$
\ddot{\mathbf{e}}-\lambda_{1} \dot{\mathbf{e}}-\lambda_{2} \mathbf{e}=0
$$

which implies that e will approach to zero exponentially in time $t$.

However, due to lack of knowledge of the system parameters, we assume $\mathbf{A}_{\mathbf{e}}$ and $\mathbf{B}_{\mathbf{e}}$ are unknown, and change Eq. (21) into

$$
\mathbf{u}=\hat{\mathbf{B}}_{\mathbf{e}}^{-1}\left(-\hat{\mathbf{A}}_{\mathbf{e}} \mathbf{e}-\lambda_{1} \dot{\mathbf{e}}-\lambda_{2} \mathbf{e}\right)
$$

by replacing $\mathbf{A}_{\mathbf{e}}$ and $\mathbf{B}_{\mathbf{e}}$ by their on-line estimates $\hat{\mathbf{A}}_{\mathbf{e}}$ and $\hat{\mathbf{B}}_{\mathbf{e}}$, respectively. Thus, the differential Eq. (22) becomes:

$$
\begin{aligned}
\ddot{\mathbf{e}} & =\mathbf{A}_{\mathbf{e}} \mathbf{e}+\mathbf{B}_{\mathbf{e}} \hat{\mathbf{B}}_{\mathbf{e}}^{-1}\left(-\hat{\mathbf{A}}_{\mathbf{e}} \mathbf{e}-\lambda_{1} \dot{\mathbf{e}}-\lambda_{2} \mathbf{e}\right) \\
& =\mathbf{A}_{\mathbf{e}} \mathbf{e}+\left(\hat{\mathbf{B}}_{\mathbf{e}}+\tilde{\mathbf{B}}_{\mathbf{e}}\right) \hat{\mathbf{B}}_{\mathbf{e}}^{-1}\left(-\hat{\mathbf{A}}_{\mathbf{e}} \mathbf{e}-\lambda_{1} \dot{\mathbf{e}}-\lambda_{2} \mathbf{e}\right)=\tilde{\mathbf{A}}_{\mathbf{e}} \mathbf{e}+\tilde{\mathbf{B}}_{\mathbf{e}} \mathbf{u}-\lambda_{1} \dot{\mathbf{e}}-\lambda_{2} \mathbf{e}
\end{aligned}
$$

where $\tilde{\mathbf{A}}_{\mathbf{e}}=\mathbf{A}_{\mathbf{e}}-\hat{\mathbf{A}}_{\mathbf{e}}$, and $\tilde{\mathbf{B}}_{\mathbf{e}}=\mathbf{B}_{\mathbf{e}}-\hat{\mathbf{B}}_{\mathbf{e}}$ are both the matrices of estimation errors.

\subsection{Self-tuning adaptive laws}

In the previous section, we have derived the differential equations of $\mathbf{e}$, which involves matrices $\tilde{\mathbf{A}}_{\mathbf{e}}$ and $\tilde{\mathbf{B}}_{\mathbf{e}}$. Now, if we design the STA laws of $\tilde{\mathbf{A}}_{\mathbf{e}}$ and $\tilde{\mathbf{B}}_{\mathbf{e}}$ as

$$
\begin{array}{r}
-\dot{\tilde{\mathbf{A}}}_{\mathrm{e}}^{T}=\dot{\hat{\mathbf{A}}}_{\mathrm{e}}^{T}=\mathbf{G}_{\mathbf{A}} \dot{e}^{\mathbf{T}}, \\
-\dot{\tilde{\mathbf{B}}}_{\mathrm{e}}^{T}=\dot{\hat{\mathbf{B}}}_{\mathrm{e}}^{T}=\mathbf{G}_{\mathbf{B}} \mathbf{u} \dot{\mathbf{e}}^{\mathbf{T}}
\end{array}
$$

where $\mathbf{G}_{\mathbf{A}}>\mathbf{0}$ and $\mathbf{G}_{\mathbf{B}}>\mathbf{0}$ are both positive definite matrixes. Apparently, the two estimates both have effects on the convergence of the errors. Then, applying the control law in Eq. (23) with the STA laws in Eqs. (25) and (26), we claim that the error e will approach $\mathbf{0}$ as $t$ tends to infinity.

\subsection{Stability analysis}

Before we proceed to prove the stability of the closed-loop system, we should define the error equation first which is necessary for the stability analysis. Let

$$
\mathbf{E}=\left[\begin{array}{llll}
\dot{e}_{1} & e_{1} & \dot{e}_{2} & e_{2}
\end{array}\right]^{T}
$$

then its time derivative can be formed as 


$$
\begin{aligned}
\dot{\mathbf{E}} & =\left[\begin{array}{l}
\ddot{e}_{1} \\
\dot{e}_{1} \\
\ddot{e}_{2} \\
\dot{e}_{2}
\end{array}\right]=\left[\begin{array}{llll}
-\lambda_{1} & -\lambda_{2} & 0 & 0 \\
1 & 0 & 0 & 0 \\
0 & 0 & -\lambda_{1} & -\lambda_{2} \\
0 & 0 & 1 & 0
\end{array}\right]\left[\begin{array}{l}
\dot{e}_{1} \\
e_{1} \\
\dot{e}_{2} \\
e_{2}
\end{array}\right]+\left[\begin{array}{llll}
0 & \tilde{a}_{21} & 0 & \tilde{a}_{23} \\
0 & 0 & 0 & 0 \\
0 & \tilde{a}_{41} & 0 & \tilde{a}_{43} \\
0 & 0 & 0 & 0
\end{array}\right] \\
& {\left[\begin{array}{l}
\dot{e}_{1} \\
e_{1} \\
\dot{e}_{2} \\
e_{2}
\end{array}\right]+\left[\begin{array}{ll}
\tilde{b}_{21} & \tilde{b}_{22} \\
0 & 0 \\
\tilde{b}_{41} & \tilde{b}_{42} \\
0 & 0
\end{array}\right]\left[\begin{array}{l}
u_{1} \\
u_{2}
\end{array}\right] } \\
& \equiv-\mathbf{L E}+\mathbf{W}_{1} \mathbf{E}+\mathbf{W}_{2} \mathbf{u} .
\end{aligned}
$$

Now, we are ready to prove the asymptotic convergence of the state vector $\mathbf{E}$, i.e., $\mathbf{E}$ approaches $\mathbf{0}$ as $t$ tends to infinity by Lyapunov stability theory [9]. To that purpose, we select a Lyapunov function candidate as:

$$
\mathbf{V}=\mathbf{V}_{1}+\mathbf{V}_{2}
$$

where

$$
\begin{aligned}
& \mathbf{V}_{1}=\frac{1}{2} \mathbf{E}^{\mathbf{T}} \mathbf{E} \\
& \mathbf{V}_{2}=\frac{1}{2} \operatorname{tr}\left(\tilde{\mathbf{A}}_{\mathbf{e}} \mathbf{G}_{\mathbf{A}}^{-1} \tilde{\mathbf{A}}_{\mathbf{e}}^{\mathbf{T}}\right)+\frac{1}{2} \operatorname{tr}\left(\tilde{\mathbf{B}}_{\mathbf{e}} \mathbf{G}_{\mathbf{B}}^{-1} \tilde{\mathbf{B}}_{\mathbf{e}}^{\mathbf{T}}\right) .
\end{aligned}
$$

Their respective time derivatives are evaluated as

$$
\begin{aligned}
& \dot{\mathbf{V}}_{1}=-\mathbf{E}^{\mathrm{T}} \mathbf{L E}+\dot{\mathbf{e}}^{\mathrm{T}} \tilde{\mathbf{A}}_{\mathbf{e}} \mathbf{e}+\dot{\mathbf{e}}^{\mathrm{T}} \tilde{\mathbf{B}}_{\mathbf{e}} \mathbf{u}, \\
& \dot{\mathbf{V}}_{2}=\operatorname{tr}\left(\tilde{\mathbf{A}}_{\mathbf{e}} \mathbf{G}_{\mathbf{A}}^{-1} \tilde{\mathbf{A}}_{\mathbf{e}}^{\mathbf{T}}\right)+\operatorname{tr}\left(\tilde{\mathbf{B}}_{\mathbf{e}} \mathbf{G}_{\mathbf{B}}^{-1} \tilde{\dot{\mathbf{B}}}_{\mathbf{e}}^{\mathbf{T}}\right)
\end{aligned}
$$

by use of Eq. (28). Obviously, we obtain two facts

$$
\mathbf{V}=\mathbf{V}_{1}+\mathbf{V}_{2} \geq 0
$$

and

$$
\begin{aligned}
& \dot{\mathbf{V}}=\dot{\mathbf{V}}_{1}+\dot{\mathbf{V}}_{2} \\
& =-\mathbf{E}^{\mathrm{T}} \mathbf{L} \mathbf{E}+\dot{\mathbf{e}}^{\mathrm{T}} \tilde{\mathbf{A}}_{\mathbf{e}} \mathbf{e}+\dot{\mathbf{e}}^{\mathrm{T}} \tilde{\mathbf{B}}_{\mathbf{e}} \mathbf{u}+\operatorname{tr}\left(\tilde{\mathbf{A}}_{\mathbf{e}} \mathbf{G}_{\mathbf{A}}^{-1} \tilde{\dot{\mathbf{A}}}\right)+\operatorname{tr}\left(\tilde{\mathbf{B}}_{\mathbf{e}} \mathbf{G}_{\mathbf{B}}^{-1} \tilde{\mathbf{B}}_{\mathbf{e}}^{\mathbf{T}}\right) \\
& =-\mathbf{E}^{\mathrm{T}} \mathbf{L E} \leq 0
\end{aligned}
$$

by applying the STA laws in Eqs. (25) and (26). 
From Eqs. (34) and (35), it shows that $\mathbf{V}$ is a suitable Lyapunov function, and, by Lyapunov stability criteria, we conclude that $\mathbf{E}, \tilde{\mathbf{A}}_{\mathbf{e}}$, and $\tilde{\mathbf{B}}_{\mathbf{e}}$ are all bounded, $\mathbf{E} \in L_{2}$, and in turn $\dot{\mathbf{E}} \in L_{\infty}$ by referring back to Eq. (28). Thus, by using Barbalat's Lemma [9], we can finally conclude $\mathbf{E}$ and hence e will tend to $\mathbf{0}$ asymptotically in time $\boldsymbol{t}$.

\section{Simulation and experiment result}

\subsection{Simulation result}

In this section, a series of simulation and experiment are proposed for the maglev system using the PD controller and the STA controller. The mass of the carrier with empty load is $643 \mathrm{~g}$, and the moment of inertia with respect to the $Z$ axis is $0.041 \mathrm{~kg} \mathrm{~m}^{2}$. The destabilizing force can be roughly measured by the spring force meter, and may be further transformed into the destabilizing force constant $K_{\mathrm{d}}$ which is equal to $243 \mathrm{~N} / \mathrm{m}$. Finally, we obtain the stabilizing force constant $K_{\mathrm{s}}=-0.23 \mathrm{~N} / \mathrm{A}$ in the nominal system. The control parameters for the PD controller are set as $K_{\mathrm{p}}=100, K_{\mathrm{D}}=10$ and those for the STA controller are set as $\lambda_{1}=10, \lambda_{2}=20, \mathbf{G}_{\mathbf{A}}=210$ and $\mathbf{G}_{\mathbf{B}}=205$.
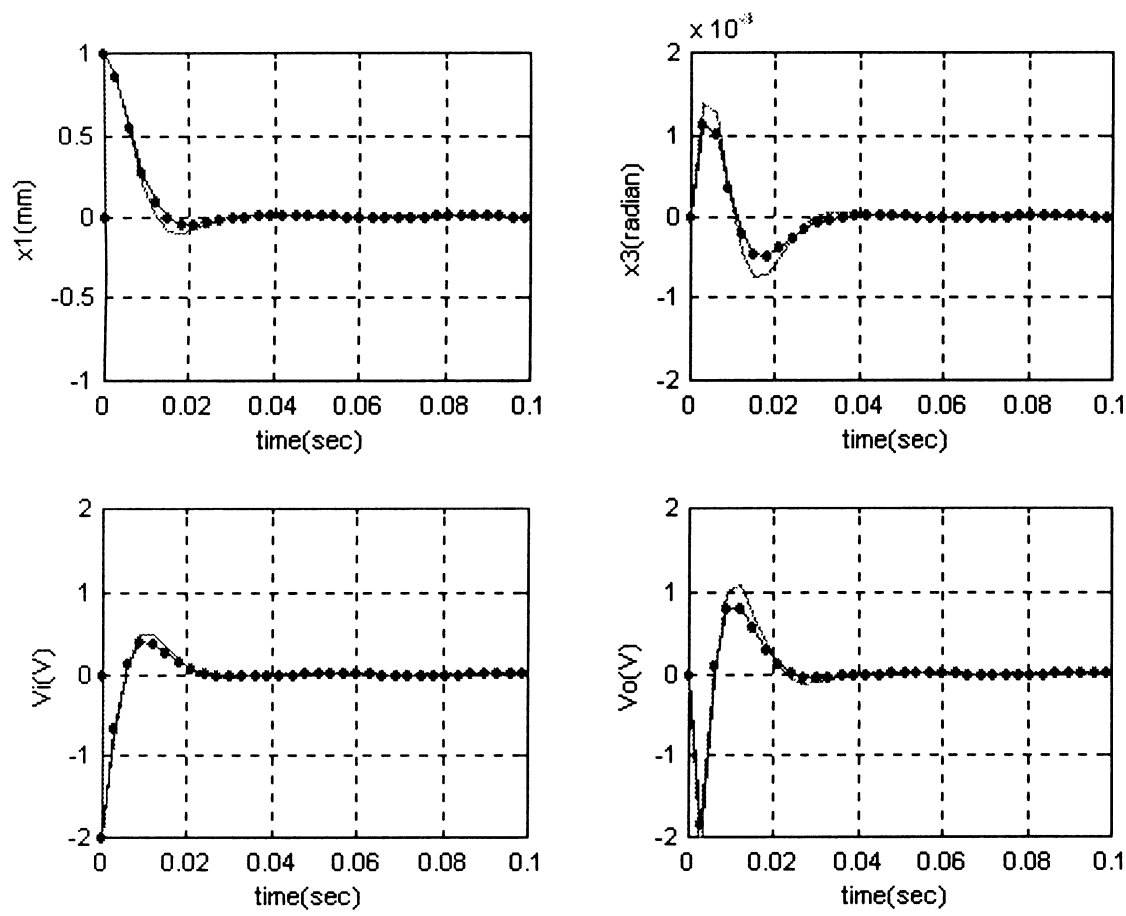

Fig. 10. Transient response of the initial condition with the maximum translational errors. 
Here, we will show the simulation results with initial conditions of the largest transitional and rotational displacements, respectively. Either one of the two situations will be encountered before the controller is turned on. The two inputs, inner control voltages $V_{\mathrm{i}}$ and outer control voltage $V_{\mathrm{o}}$, as well as the two output signals, the $\times 1$ (translational displacement) and $\times 3$ (rotational displacement) are shown in Figs. 10 and 11.

Fig. 10 shows the transient response of the largest translational displacement, i.e., $+1 \mathrm{~mm}$ for $\times 1$ in our mechanical system. When the carrier undergoes its largest translational error, the corresponding rotational error must be zero in an ideal case, and the directions of the two control forces are always the same as we have expected.

The same results can be obtained in the case of the transient response for the largest rotational displacement, 0.01 radian for $\times 3$ in our system, which is shown in Fig. 11. The opposite forces are experienced on the inner and outer magnets to generate an anti-rotational torque, which is also coincident with our expectation.

By these simulation results, we can observe that all of the four signals converge to zero eventually as $t$ goes to infinity, which means a zero power consumption is possible by such a system design. Furthermore, the PD control and STA control almost have the same performance.
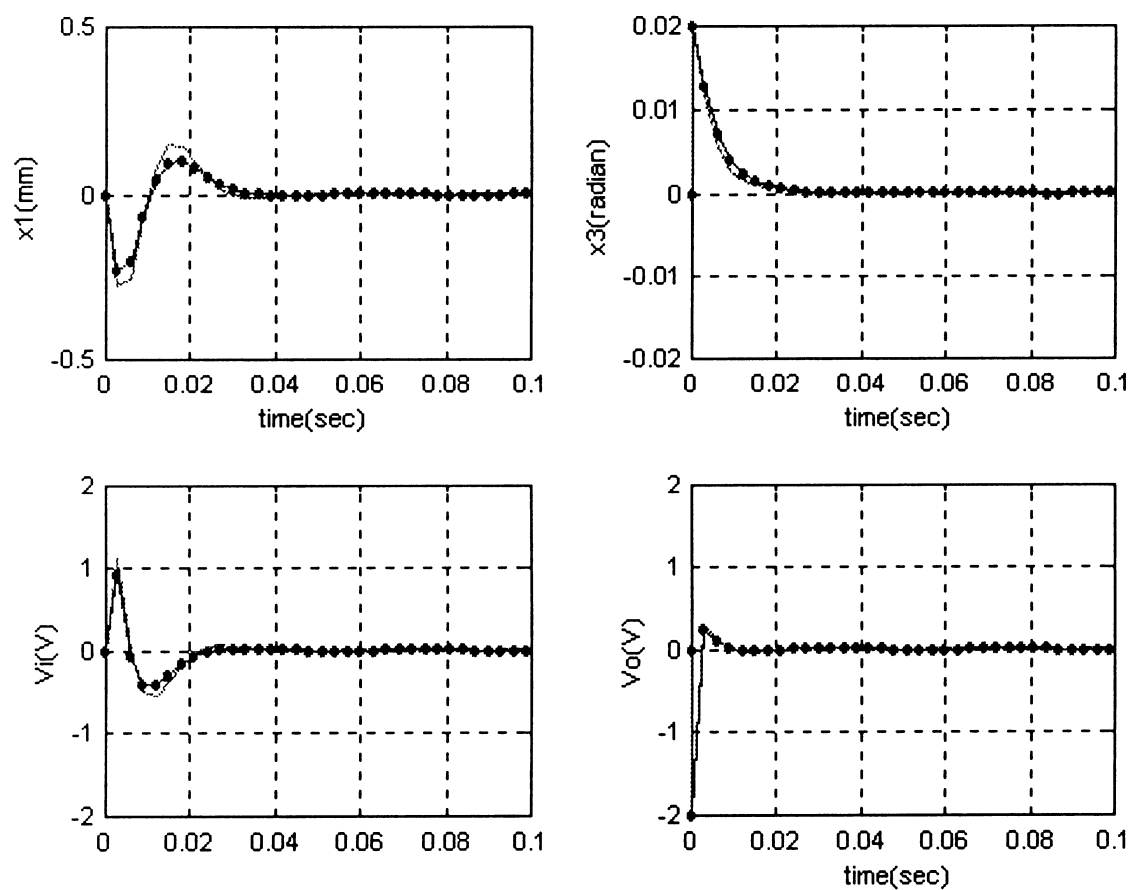

Fig. 11. Transient response of the initial condition with the maximum rotational errors (- is PD control, $-\cdot-$ is STA control). 
However, almost all the current parameters we used are given rather roughly by using some simple processes. Moreover, a change of the load will unfortunately cause the change to all the system parameters. Therefore, the above simulation results may well demonstrate the feasibility of our controller design as well as the consistent physical behavior. In the next section, the tendencies of these simulation results will be used to examine the later experimental results.

\subsection{Experimental system setup}

Fig. 12 is the block diagram of the closed-loop system, and Fig. 13 is the photograph of the overall system hardware. In the photograph, we can see the entire maglev system in which the floating table carries a 6-inch wafer, and the two sets of sensing systems are mounted at the two ends of the levitation tracks.

\subsection{Experimental results}

In the controlled situation, without the anti-gravitational forces from the levitation coils, the carrier can support a load up to $250 \mathrm{~g}$ weight. However, due to the inevitable manufacturing errors of the carrier as well as the differences in magnetic characteristics of the four $\mathrm{NdFeB}$ and rubber magnets, some adjustment of the currents in the four levitation coils are still necessary in order to form a precise horizontal plane.

Due to lack of precision equipment for calibration, we currently let the neutral location of the carrier be the mid-points of its rotational and translational ranges in our experiments. In fact, the mismatch between the defined force balance point $(X=0)$ and the natural force balance $(x=0)$ are almost inevitable. From Fig. 14 , the magnet experiences a destabilizing force when the carrier is exactly controlled at the defined force balance point $(x=0)$, which implies that it is not an equilibrium point. A steady-state error does exist by using the proposed controller in such a case. Therefore, an additional tuning component through STA

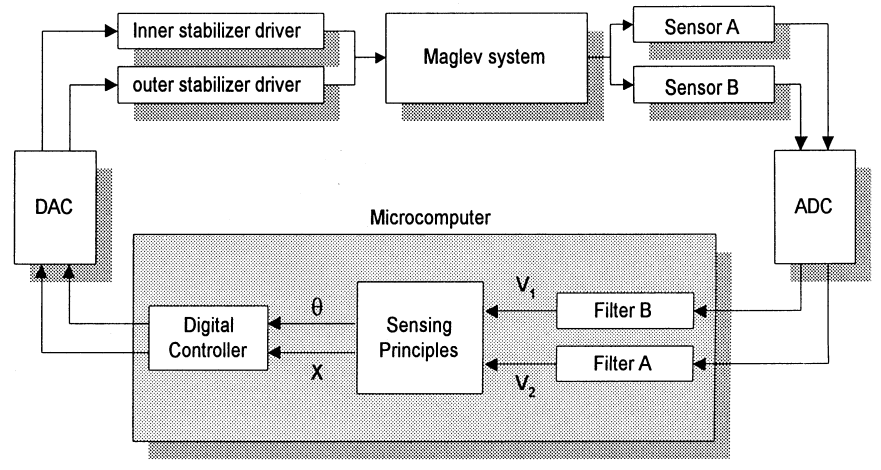

Fig. 12. Block diagram of the closed loop system. 


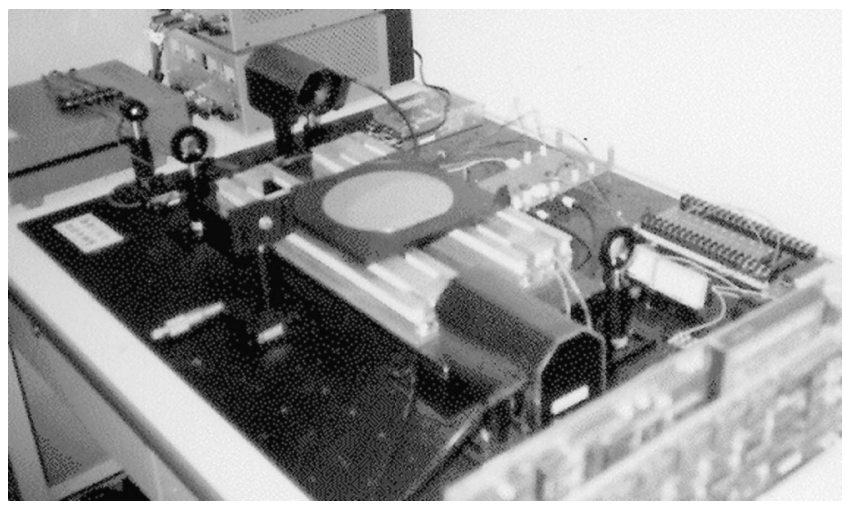

Fig. 13. Photograph of an aspect of the physical system.

control is necessary to generate a corresponding stabilizing force to reduce the undesirable steady-state error in the digital implementation.

Now, the transient as well as steady-state responses will be investigated with empty load or $250 \mathrm{~g}$ load, given the two possible initial conditions as shown in Fig. 6, namely, initial maximum translational displacement and initial maximum rotational displacement. Each case is demonstrated with four time trajectories for two inputs and two outputs. From these figures, the positioning accuracy of the levitated carrier can be readily determined by the steady-state resonance level. In our experimental result, the sampling time is chosen from 0.1 to $0.05 \mathrm{~ms}$ so that this system can be better behaved.

\subsubsection{Transient response}

Before we investigate the transient responses of the closed-loop system, some

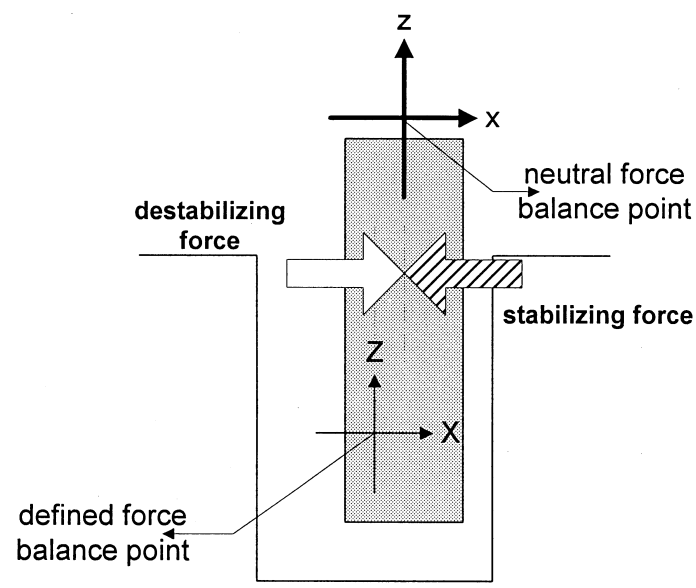

Fig. 14. Mismatch of the neutral point. 
specifications should be revealed. First, the full $x$-direction translation range is limited to $2 \mathrm{~mm}$, or $\pm 1 \mathrm{~mm}$, whereas the $z$-axis full rotational range is 0.4 radian, or \pm 0.2 radian. The state variable of the former is assigned to be $\times 1$ in controller design, whereas that of the latter, i.e., $\theta$, is assigned to be $\times 3$. Next, the trajectories of the other two input control voltages labeled with $V_{\mathrm{i}}$ and $V_{\mathrm{o}}$ are both limited to $\pm 5 \mathrm{~V}$ due to the setting of $\mathrm{D} / \mathrm{A}$ converters. On the other hand, we may compare the performances of the two cases respectively with empty load and 250 g load.

In Figs. 15-16 and Figs. 18-19, respectively, we illustrate the transient responses for the case with initial maximum translational error and for that with the initial maximum rotational mis-displacement. From the trajectories of $\times 1$ and $\times 3$, one can see that both of the two error signals converge to their steady states within about $0.2 \mathrm{~s}$. The wave forms of the two control inputs, $V_{\mathrm{i}}$ and $V_{\mathrm{o}}$, are about the same as the corresponding simulation results. However, an obvious difference exists in the control efforts. When the carrier is with $250 \mathrm{~g}$ load, the control voltages are larger than those in the case with the empty load. Although both the PD controller and STA controller can also successfully control the maglev system to some extent of precision following a similar trend of transient response, there are significant steady-state errors for the PD controller, especially when the payload is $250 \mathrm{~g}$. This is exactly the salient feature of our STA controller, which may adjust its controller on-line to adapt to the change in the environment to ensure the system performance.

In theory, the control inputs should eventually converge to zero when the
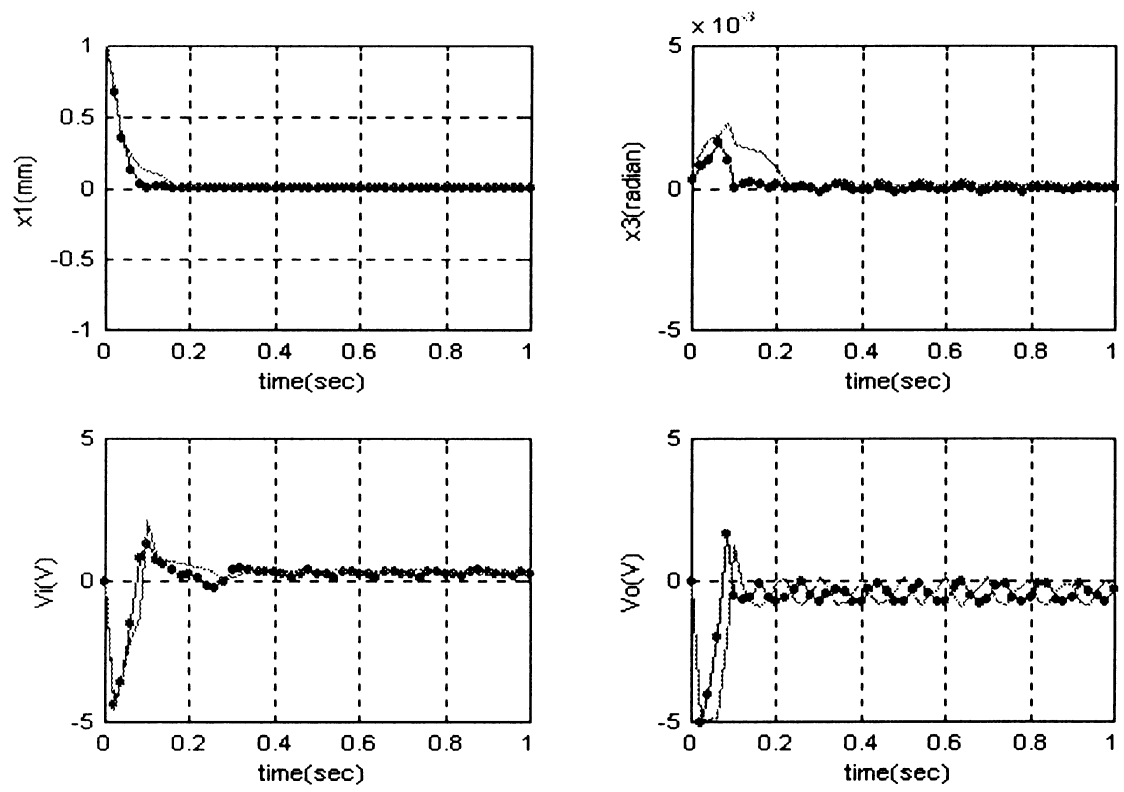

Fig. 15. Transient response w.r.t. the largest positive translational displacement with empty load. 

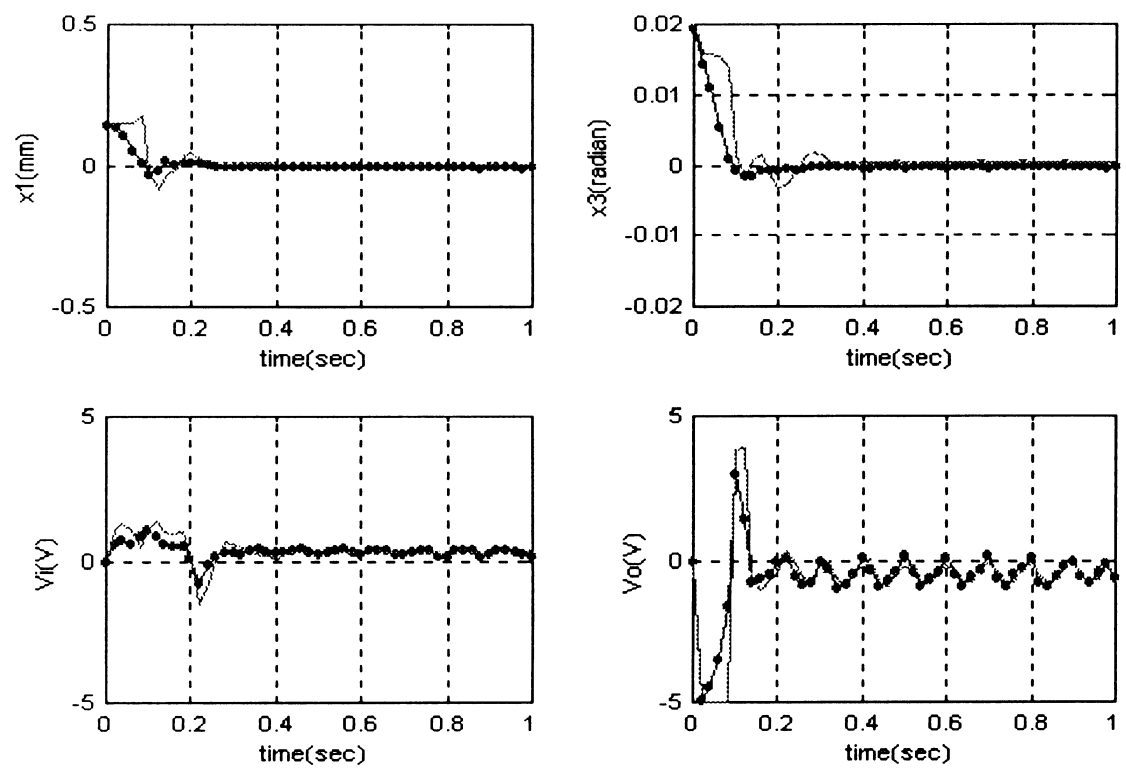

Fig. 16. Transient response w.r.t. the largest positive rotational displacement with empty load.
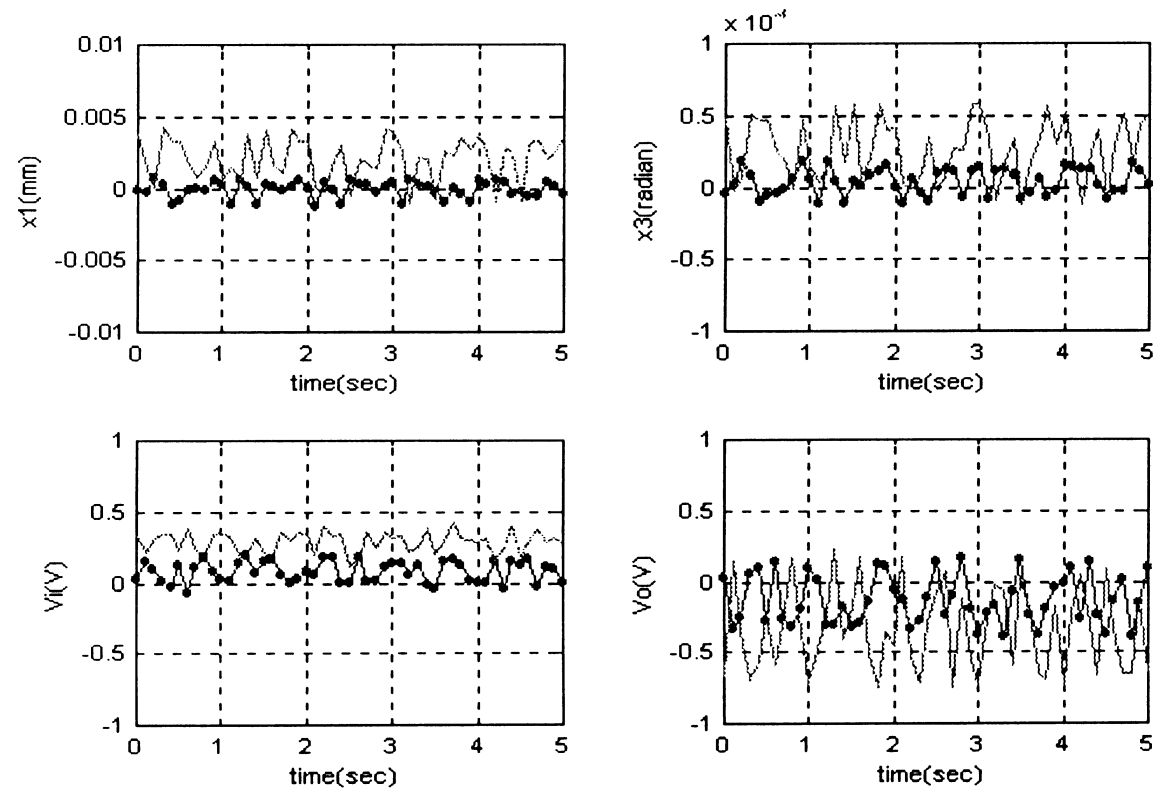

Fig. 17. Steady-state response with empty load. 

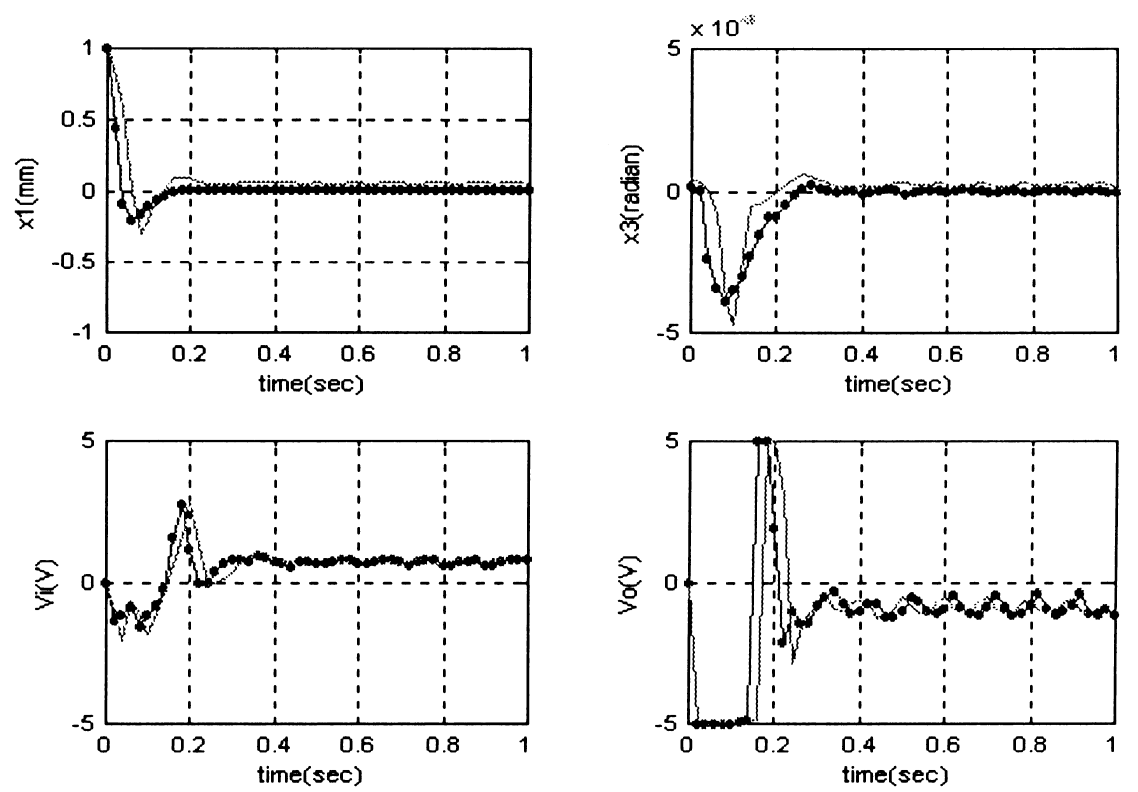

Fig. 18. Transient response w.r.t. the largest positive translational displacement with $250 \mathrm{~g}$ load.
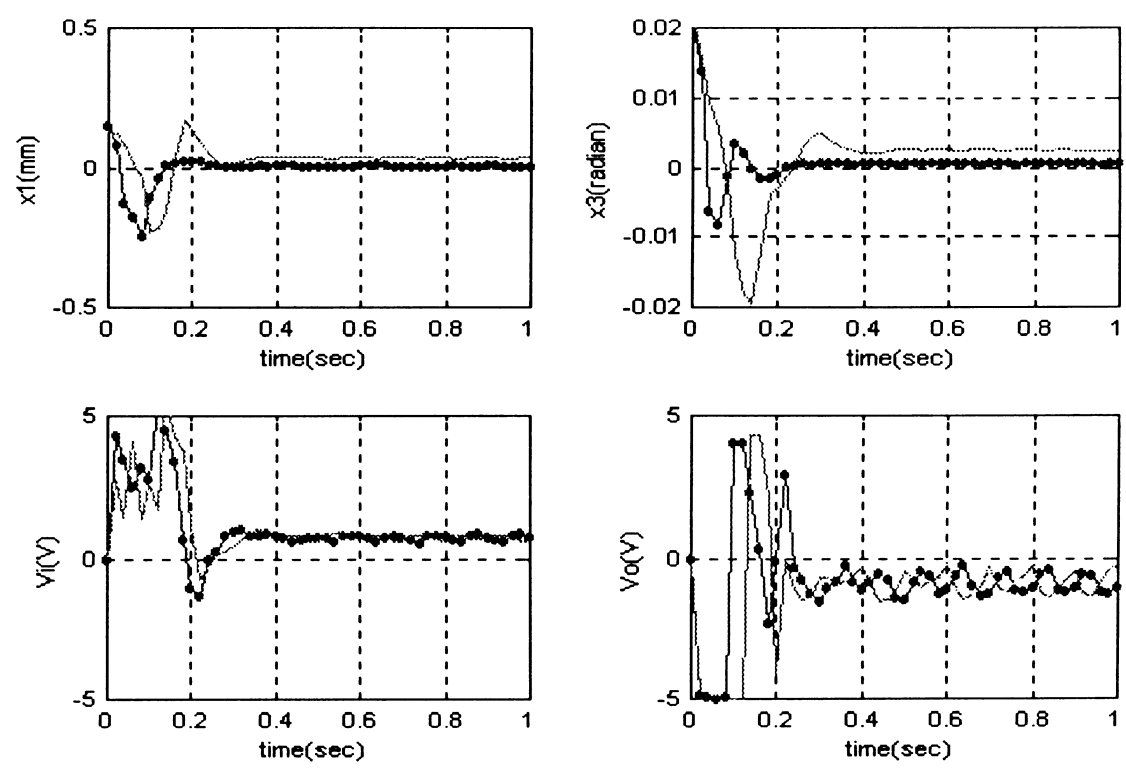

Fig. 19. Transient response w.r.t. the largest positive rotational displacement with $250 \mathrm{~g}$ load. 
steady state is reached. However, in our experimental results, it is a fact that both of the two control inputs have their biases. In other words, only when the central lines of tracks are coincident with those of the two laser beams, zero mean of the two control inputs can be possible.

\subsubsection{Steady-state response}

The positioning precision of our system is determined by the steady-state response of the carrier. Figs. 17 and 20 show the system responses around the operating points with respect to the empty load and the $250 \mathrm{~g}$ load. The PD controller can provide the final precision level up to $6 \mu \mathrm{m}$ in translation and $1.5 \times 10^{-3}$ radian in rotation. However, the STA controller can reach the final precision level up to $4 \mu \mathrm{m}$ in translation and $9 \times 10^{-4}$ radian in rotation. These results show that the STA controller is superior in its regulating performance, even when the carrier load varies. In conclusion, the entire performance of the STA controller is better than that of the PD controller under similar control parameters.

It is obvious that the resulting time responses are combinations of a low frequency component and some high frequency ones. The low frequency component, which is around $0.5 \mathrm{~Hz}$, arises from the vibration of the surrounding environment, while the other frequency components are possibly due to the operation of electromechanical system and interference of the optical sensors.
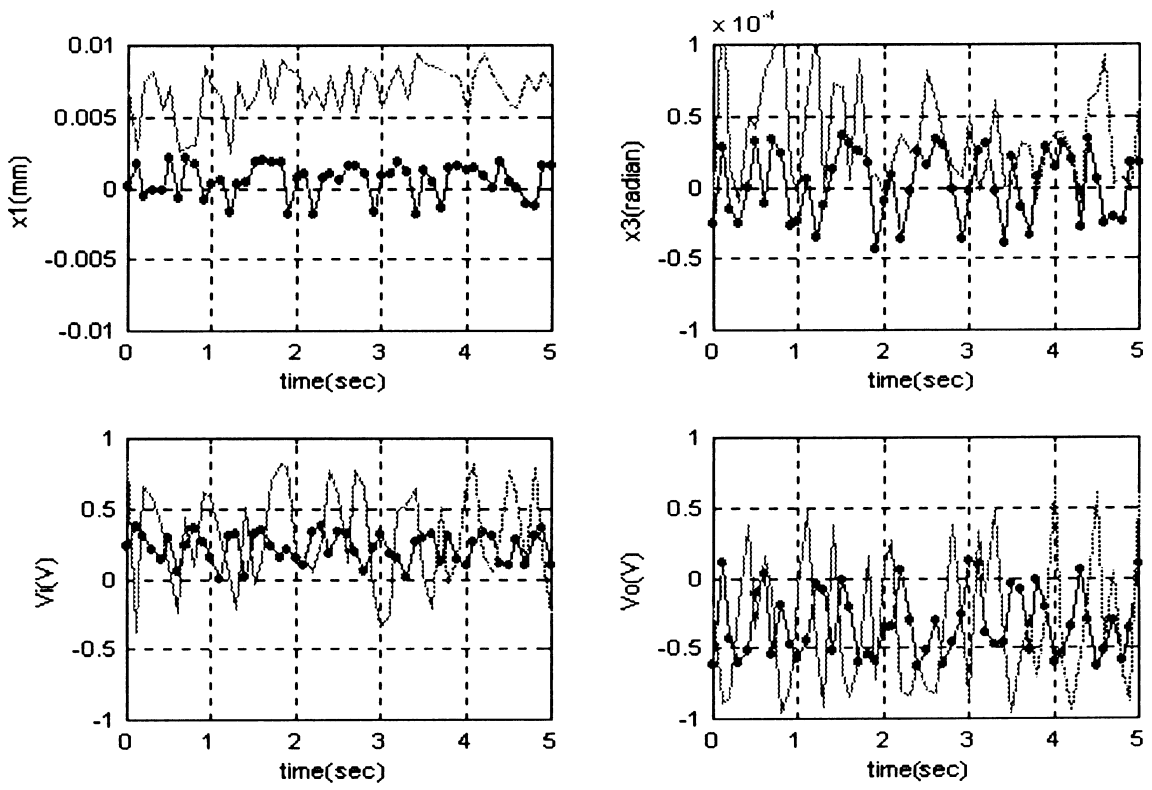

Fig. 20. Steady-state response with $250 \mathrm{~g}$ load. 


\subsection{Discussion}

Besides the manufacturing and alignment imperfections, which may affect the transient responses, the vibrations from the interior of the building or from the mechanism, and the optical interference may affect the steady-state responses. Other than these, there are some important factors, such as edge effect and damping force.

By the experiment, the hereby developed system which applied STA control, obtains performance better than that of [8]. The advantage of the STA controller is that even if the static term is not known exactly, the tuning process will modify the static term to its true value and will result in improved performance. A comparison between our system and that in [8] is summarized in Table 2.

\section{Conclusions}

In this research, a short-range travel maglev with high precision is designed. A prototype repulsive maglev system with four active guiding tracks was developed here by applying the advanced technologies in magnetic materials, microelectronics, and optical sensors. For the purpose of stabilizing the carrier, the system is treated as a 2-input 2-output system, and an STA controller is designed and implemented by using a microcomputer. From the simulation and experimental results, the system's feasibility and effectiveness have been clearly demonstrated. A detailed structure comparison between the system developed in this paper and that in [8] was also made. It has been shown that the performance here in general is superior to that of [8], for the current system design is an improved version of that in [8] and a better control strategy was proposed. On the other hand, the regulating performance shows that the effects due to manufacturing and alignment imperfections are eliminated completely. This implies that the improvement of the performance is possible by use of a more delicate control rather than a more expensive hardware setup.

Table 2

The comparison between the hereby developed system and the system developed in [8]

\begin{tabular}{lll}
\hline Property & Wang's original system & Hereby developed system \\
\hline Levitation magnets & Electromagnets & Hybrid magnets \\
Loading capacity & 150 g (fixed) & 1000 g (variable) \\
Power consumption & High & Low \\
Efficiency & Low & High \\
Controller hardware & Analog circuit & Digital microprocessor \\
Control algorithm & Pole placement control & STA control \\
Flexibility & Poor & Good \\
Laser beams & Divergent & Collimated \\
Sensor gains & Varying \& nonlinear & Fixed \& linear \\
\hline
\end{tabular}


Finally, by using permanent magnets here, a nearly zero power guiding system has been realized. With the same size, the low energy dissipation and high load capacity of about $1 \mathrm{~kg}$ were major advantages of such a design which are much better than that of the related research results so far to our knowledge.

\section{References}

[1] Hollis RL, Salcudean SE. On the design and control of magnetically levitated robot wrists, robotics and manufacturing, recent trends in research, education and application. In: Proceedings of the 3rd International Symposium on Robotis and Manufact. New York: ASME Press, 1990.

[2] Hollis RL, Salcudeam SE. Six degree of freedom magnetically levitated variable compliance fine motion wrist. In: Proceedings of the 4th International Symposium on Robotics Research, Santa Cruz Ca. Aug. 1987, in Robotis Research. MIT Press, 1987. p. 4.

[3] Jayawant BV, Sinha PK, Aylwin DG. Feedback control system for D.C. electromagnets in passenger-carrying vehicles. Int J Control 1976;24(5):627-39.

[4] Proise M. System concept definition of the Grumman superconducting electromagnetic suspension (EMS) maglev design. In: Maglev '93 Conference Argonne National Laboratory, 19-21 May, 1993.

[5] Covert EE, Vlajinac M, Stephens T, Finston M. Magnetic balance and suspension systems for use with wind tunnels. In: Kuchemann D, editor. Progress in aerospace science, vol. 14. Pergamon Press, 1973. p. 27-107.

[6] Higuchi T. Magnetic bearings. In: Proceedings of the 1st International Symposium on Magnetic Bearings, University of Tokyo, 1990.

[7] Kosuke N, Masashi I. A noncontact permanent magnet levitation table with electromagnetic control and its vibration isolation method using direct disturbance cancellation combining optimal regulators. IEEE Tran on Magnetics 1995;31(1).

[8] Wang IY. A magnetic levitation silicon wafter transport system. PhD Thesis, The University of Texas at Austin, 1993.

[9] Narendra KS, Annaswamy AM. Stable adaptive system. Prentice Hall, Inc. A. Simon and Schuster Company Englewood Cliffs, New Jersey, 1989. 